\title{
Feasibility of non-exposed endoscopic wall-inversion surgery with sentinel node basin dissection as a new surgical method for early gastric cancer: a porcine survival study
}

\author{
Osamu Goto $\cdot$ Hiroya Takeuchi $\cdot$ Hirofumi Kawakubo $\cdot$ Satoru Matsuda $\cdot$ \\ Fumihiko Kato $\cdot$ Motoki Sasaki $\cdot$ Ai Fujimoto $\cdot$ Yasutoshi Ochiai $\cdot$ \\ Joichiro Horii · Toshio Uraoka $\cdot$ Yuko Kitagawa $\cdot$ Naohisa Yahagi
}

Received: 13 December 2013/Accepted: 13 February 2014/Published online: 12 March 2014

(c) The International Gastric Cancer Association and The Japanese Gastric Cancer Association 2014

\begin{abstract}
Non-exposed endoscopic wall-inversion surgery (NEWS) has been developed as an endoscopic fullthickness resection technique without translumenal communication to avoid intraabdominal infection or tumor seeding. We aimed to investigate the feasibility and safety of NEWS with sentinel node basin dissection (SNBD), which can minimize the area of lymphadenectomy for early gastric cancer (EGC), in 10 porcine survival models. After placing laparoscopic ports and making markings on both the mucosal and serosal sides of a simulated lesion, indocyanine green fluid was endoscopically injected into the submucosa at 4 quadrants around the lesion. An SN basin including the stained SNs was dissected, and a circumferential sero-muscular incision around the lesion and seromuscular suturing were performed laparoscopically, with the lesion inverted toward the inside of the stomach. Finally, circumferential mucosal incision and transoral retrieval were made endoscopically. In all cases, the lesion was resected in an en bloc fashion, and all pigs survived without adverse events. After 1 week of observation, pigs were sacrificed for macroscopic investigation. The average procedural duration was $170 \mathrm{~min}$ (range 130-253 min). Intraoperative perforation occurred in 1 case, which could be safely treated by laparoscopic suturing. The number of
\end{abstract}

O. Goto $(\bowtie) \cdot$ M. Sasaki · A. Fujimoto · Y. Ochiai · J. Horii · T. Uraoka $\cdot$ N. Yahagi

Division of Research and Development for Minimally Invasive Treatment, Cancer Center, Keio University, School of Medicine, 35 Shinanomachi, Shinjuku-ku, Tokyo 160-8582, Japan

e-mail: ogotou-gi@a3.keio.jp

H. Takeuchi $\cdot$ H. Kawakubo $\cdot$ S. Matsuda $\cdot$ F. Kato ·

Y. Kitagawa

Department of Surgery, Keio University, School of Medicine,

Tokyo, Japan dissected SN basins was 1 in 9 cases and 2 in 1 case. Necropsy revealed no signs of severe complication. This animal survival study demonstrated that NEWS with SNBD was safe and feasible. It may provide patients with possibly node-positive EGC a minimally-sized local resection and minimally-ranged lymphadenectomy without the risk of tumor dissemination.

Keywords Minimally invasive surgery $\cdot$ Partial resection $\cdot$ Sentinel node navigation surgery $\cdot$ Early gastric cancer $\cdot$ Animal study

\section{Introduction}

Endoscopic resection as represented by endoscopic submucosal dissection (ESD) has been widely accepted as a curative treatment method for node-negative early gastric cancer (EGC) [1-5]. On the other hand, gastrectomy with standardized lymphadenectomy is necessary for possibly node-positive EGC in the current situation [6]. As one promising option of function-preserving surgeries, local resection navigated by sentinel node ( $\mathrm{SN}$ ) mapping is introduced [7, 8]. In this theory, if no metastasis to SN identified by a radiolabeled material or a dye is revealed during the operation, the area of lymphadenectomy can be minimized. Therefore, partial or segmental resection with SN basin dissection as minimally-ranged lymphadenectomy is expected to avoid wide resection of the stomach. However, these resection options for the primary site are still inadequate to minimize the resection area of the gastric wall as much as possible because a tumor margin cannot be visualized from the serosal side.

Several local resection techniques using a flexible endoscope have been introduced in order to minimize the 
resection area by confirming the border of the lesion [911]. These techniques, however, necessitate intentional perforation during the procedure, which can raise a possibility of tumor dissemination if neoplastic cells of the lesion are exposed. Accordingly, these methods are limited to gastric submucosal tumors (SMTs) without ulcer formation and are contraindicated to cancers.

Non-exposed endoscopic wall-inversion surgery (NEWS) has been developed as full-thickness resection without translumenal communication [12-14]. Due to the advantage of no risk of intraabdominal infection, NEWS has already been clinically introduced for gastric SMTs at selected hospitals. Furthermore, this promising technique can be applied to possibly node-negative EGC which is difficult to resect by ESD, as suggested in the first report of NEWS [12].

We expect that an ideal function-preserving surgery can be realized for possibly node-positive EGC by fusing NEWS with SN navigation surgery (SNNS). Therefore, we aimed to investigate the feasibility and safety of NEWS with SN basin dissection (SNBD) in a porcine survival study.

\section{Materials and methods}

\section{Preparation}

After approval from the institutional review board of the Laboratory Animal Center at Keio University School of Medicine (IRB no. 12076-0) in terms of both potential scientific contribution and ethics in animal protection, a survival experiment of NEWS with SNBD was performed on ten female pigs weighing approximately $25-30 \mathrm{~kg}$ each in the animal research facility at Keio University, School of Medicine. Surgery was executed under general anesthesia induced by intramuscular administration of midazolam $(0.2 \mathrm{mg} / \mathrm{kg})$, medetomidine $(0.1 \mathrm{mg} / \mathrm{kg})$, and atropine sulfate $(0.02 \mathrm{mg} / \mathrm{kg})$, and maintained by inhalation of isoflurane. $0.5 \mathrm{~g}$ of cefazolin sodium hydrate was injected intramuscularly just before the procedure.

\section{Procedure of NEWS with SNBD}

The experiment was conducted using ten pigs, each of which had one simulated lesion. Ten lesions were located on the anterior wall, posterior wall, and greater curvature in the upper, middle, and lower third, and on the lesser curvature in the middle third of the stomach, respectively. Although the lesions were created to cover almost all parts of the stomach in this study, we made only one lesion in the middle third of the stomach as a representative on the lesser curvature because the lesser curvature was too short and bent too much to create three lesions due to the innate characteristic of the porcine stomach compared to the human one. After setting a pig in a spine position, one camera port and four trocars were inserted. Mucosal markings were placed around the simulated lesion approximately $4 \mathrm{~cm}$ in diameter using a tip of Dual knife (KD-650L; Olympus Medical Systems, Co., Ltd., Tokyo, Japan). Subsequently, the Dual knife was pushed deeper into the gastric wall by the endoscopist so that the location could be recognized from outside of the stomach by the laparoscopic surgeon, who then placed serosal markings using a spatula-type electrode (A6284; Olympus). The location of each marking was also confirmed by a transparently visible shadow of the device from the opposite side. In case of difficulty in placing serosal markings due to the existence of an $\mathrm{SN}$ basin, the markings were made after the $\mathrm{SN}$ basin dissection. After creating the lesion, $0.5 \mathrm{ml}$ of $5 \mathrm{mg} / \mathrm{ml}$ indocyanine green (ICG) fluid solution was endoscopically injected into the submucosa at four quadrants around the lesion (Fig. 1a). SNs stained with ICG were identified approximately $10 \mathrm{~min}$ after injection, and an SN basin including the stained SNs was dissected laparoscopically using the electrode, or SonoSurg (Olympus). SN basins were divided into five parts according to the distribution of feeding arteries: right and left gastroepiploic artery (GEA), right, left, and posterior gastric artery (GA) [8]. Subsequently, circumferential sero-muscular incisions were made approximately $5 \mathrm{~mm}$ outside the serosal markings with the spatula-type electrode or the SonoSurg, and sero-muscular continuous suturing was performed, with the lesion inverted toward the inside of the stomach (Fig. 1b-d). To facilitate the subsequent endoscopic procedure, a formed polyurethane elastomer sheet (Kyowagiken Co., Ltd., Saitama, Japan), optimally cut according to the lesion size, was inserted between the serosal surface of the inverted lesion and the sutured layer on the way of suturing (Fig. 1c). Finally, a circumferential mucosal incision was made with the Dual Knife (Fig. 1e-h), and the lesion as well as the sheet was retrieved transorally. The procedure was finished by removing the port and trocars and suturing abdominal incisions.

Postoperative procedures and outcome measures

After awaking from anesthesia, the pigs were allowed to drink water, and started to feed on the next day of operation. $0.5 \mathrm{~g}$ of cefalexin was given with diet until the third day after the procedure. Pigs were sacrificed on the seventh day for macroscopic investigation of the abdominal cavity and the stomach.

As the primary outcome measure, survival rate in the observational period was investigated. As secondary outcome measures, technical feasibility, complications, and gross findings on necropsy were assessed. 

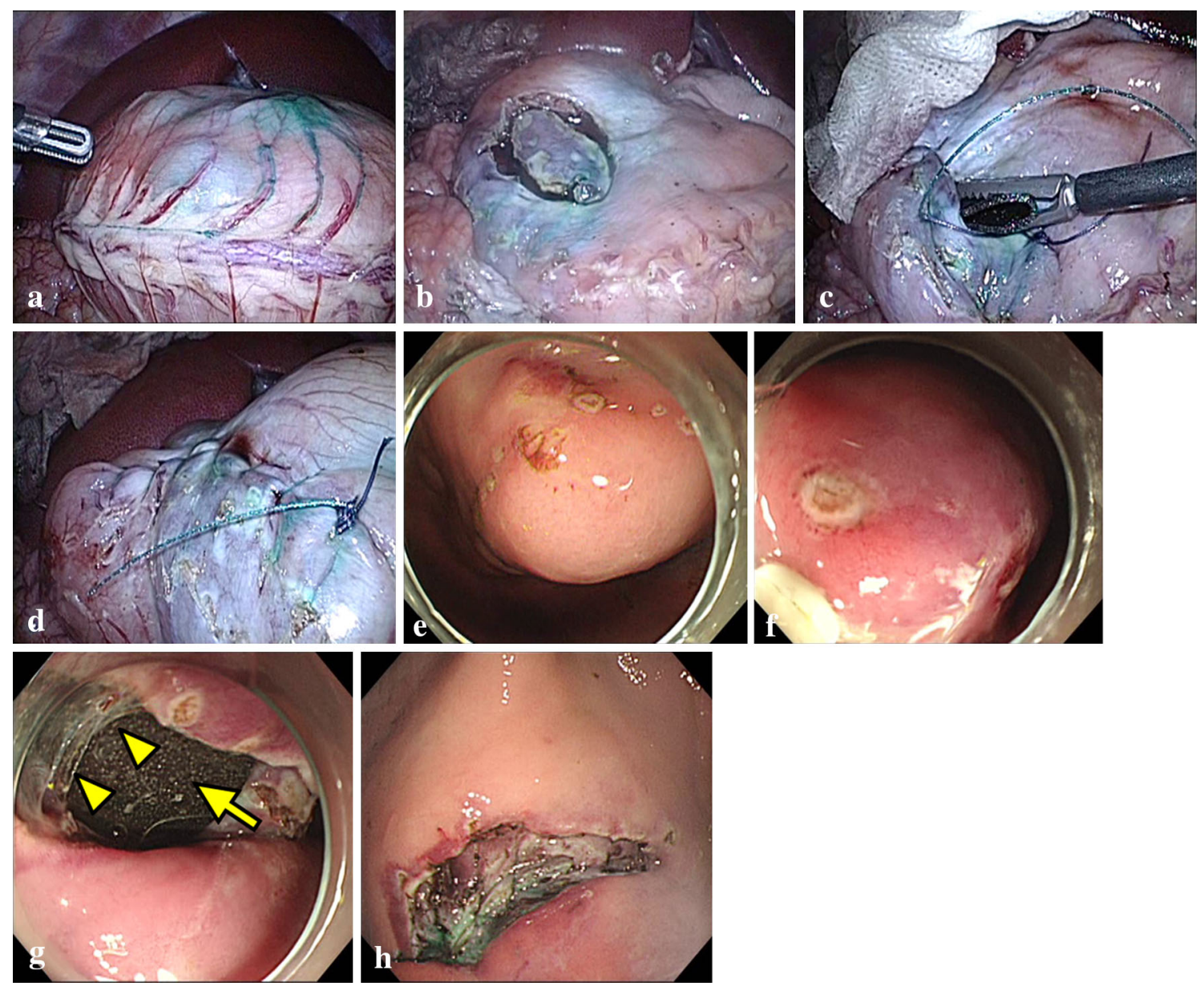

Fig. 1 Procedure of NEWS with SNBD (Case No. 5). a A lymphatic flow is visualized after submucosal fluid injection of indocyanine green around the simulating lesion. b Circumferential sero-muscular incision. c A formed polyurethane elastomer sheet optimally cut according to the size of the lesion is inserted between the isolated serosal plane and the sutured layer. d Sero-muscular continuous

\section{Results}

All pigs survived surgery without adverse events (10/10, $100 \%$ ). The full-layered lesion was resected in en bloc fashion in all cases (Fig. 2a, b). The average size of the specimen was $41 \mathrm{~mm}$ (range 25-60 mm), and the average procedural duration was $170 \mathrm{~min}$ (range 130-253 min). In one case on the lesser curvature, perforation at the phase of sero-muscular incision and incision of the suture at the phase of mucosal incision occurred, which could be safely treated by laparoscopic suturing. In another case, the sheet was unexpectedly sutured at the end of the sero-muscular suturing, which was resolved by opening the anastomotic site and suturing the full layers again after retrieving the specimen and the sheet. SN basins were dissected as follows: right GEA in five cases, left GEA in two, right GA in suturing with the lesion inverted toward the inside. e Endoscopic view of the inverted lesion. $\mathbf{f}$ A circumferential mucosal incision is made by confirming the mucosal markings. $g$ Endoscopic view during the mucosal incision. A formed polyurethane elastomer sheet (arrow) beneath the serosal place and a remnant submucosal tissue (arrowhead) are seen. h Full-thickness defect

two, left GA in one, posterior GA in one (two basins were dissected in one case), respectively (Fig. 2c).

The average weight change in the observational period was $+1.3 \mathrm{~kg}$ (range -0.4 to $+3.3 \mathrm{~kg}$ ). Necropsy revealed mild adhesion of the suture site to surrounding organs in all cases, but no signs of severe complication, e.g., anastomotic leak, necrosis of the gastric wall, or peritonitis were shown (Fig. 2d-f). In two cases, a small amount of lightly yellowish, serous ascites was collected, which was suspected mild lymphorrhea. The results are shown in Table 1.

\section{Discussion}

This animal survival study demonstrated that NEWS with SNBD was safe and feasible. Technical feasibility of 

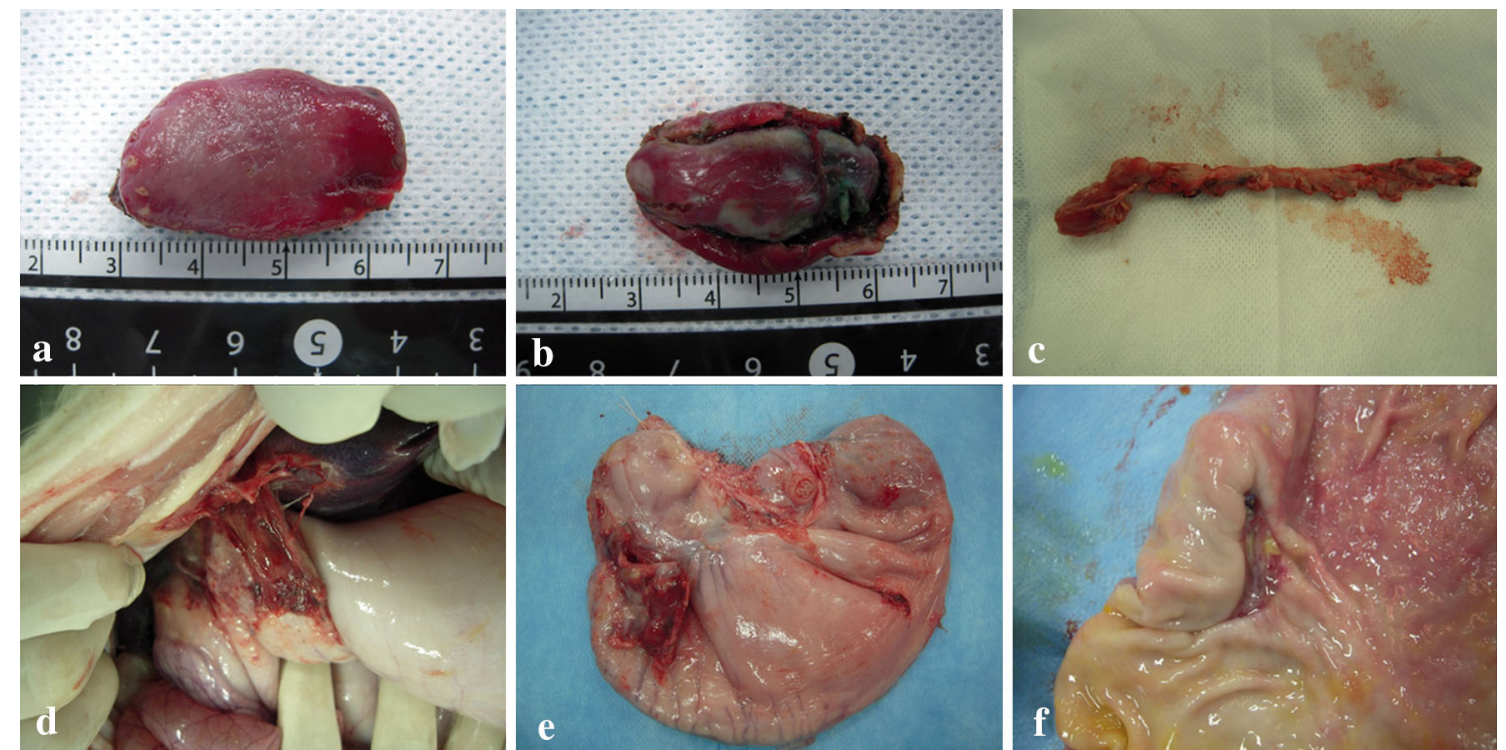

Fig. 2 Resected specimens and gross findings on necropsy (Case No. 5). a Mucosal side of the resected lesion. b Serosal side. c Dissected sentinel node basin (right gastroepiploic artery). d Mild adhesion of the suture site to the abdominal wall. e Overview of the remnant stomach. f Mucosal side of the resected area

absolutely indispensable, a spacer such as the elastomer sheet would be very helpful to facilitate the endoscopic procedure.

Mitsui and colleagues attempted endoscopic clipping after the resection in clinical cases [14], whereas we omitted it in this study. Although the effectiveness of placing endoscopic clips in the NEWS procedure is unknown, mucosal closure using clips can be of some help to avoid anastomotic leakage or postoperative bleeding. Because we should carefully and safely introduce the new technique to human cases, it would be better to perform endoscopic clipping, at least while NEWS is thought to be a new technique.

If NEWS with standard regional lymphadenectomy was performed for possibly node-positive cancers, a remnant stomach might be necrotized because the standard lymphadenectomy requires multiple removal of feeding arteries. Therefore, NEWS for possibly node-positive EGCs must be accompanied with minimally-ranged lymph node dissection. At the current moment, we consider that NEWS should be applied when one SN basin is to be dissected. Although one case in which two SN basins were dissected survived without complications in this study (Case No. 3), further investigation would be required to expand the indication of NEWS with SNBD for the patient's safety.

There are some reports regarding feasibility of ESD with lymphadenectomy for possibly node-positive EGC [15, 16]. However, cancers massively invading into the submucosa, which is a representative of cancers having a risk of lymph node metastasis, might be resected with a vertical margin positive by ESD. In this situation, ESD with lymphadenectomy cannot be regarded as a radical surgery. be a milestone to find that space. Although it may not be 


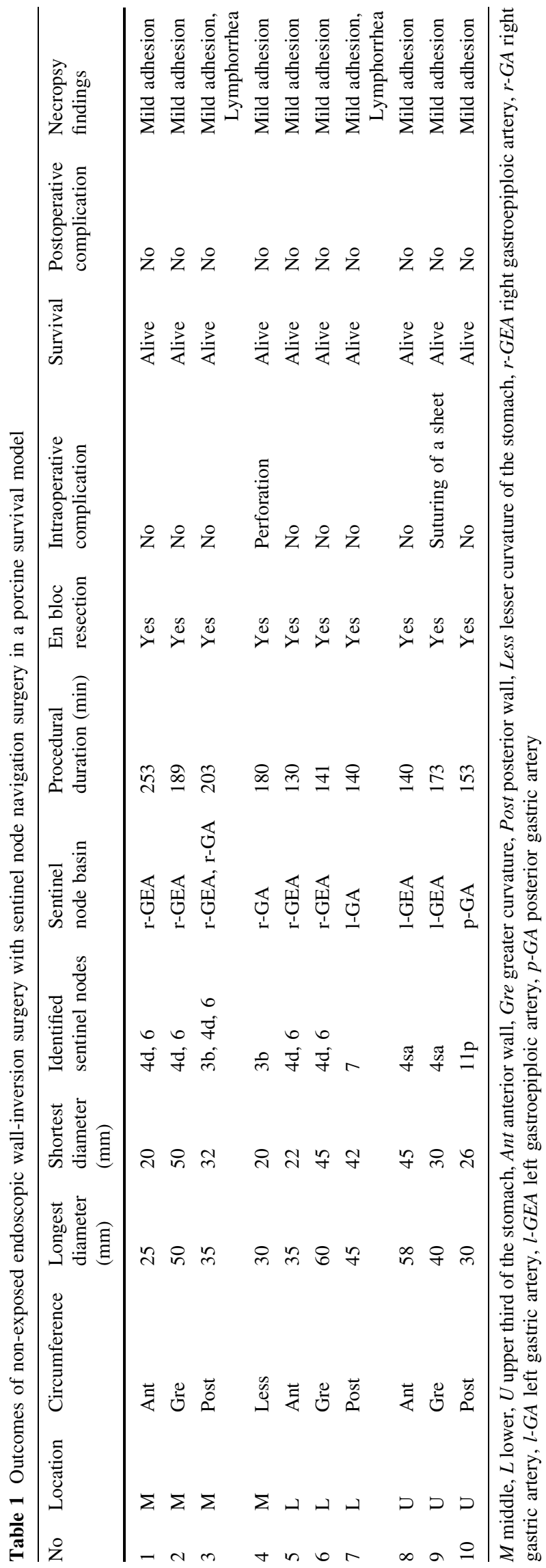

Basically, submucosal cancers should be removed on a full-thickness condition in order to obtain en bloc complete resection.

One concern is the risk of recurrence from lymphatic vessels around the primary lesion, although the probability of survival of cancer cells in lymphatic vessels would be quite low. In a clinical situation, it might be better to add the dissection of the sero-muscular layer where lymphatic vessels are stained, followed by sero-muscular suturing.

Inoue and colleagues introduced CLEAN-NET as another promising non-exposure method [17]. In this procedure, a lesion is laparoscopically pulled toward the outside of the stomach after circumferential sero-muscular incision, and is finally resected using a linear stapler. Unlike NEWS, sero-muscular hand suturing and circumferential mucosal incision can be skipped in CLEAN-NET, which will have a shorter procedural time than NEWS. In this method, however, the resection area of the gastric wall cannot be satisfactorily minimized because a mucosal border of the tumor cannot be confirmed at this step of the resection. Therefore, wide resection is always necessary to avoid a tumor-positive margin of the specimen, which may result in unacceptable deformity of the residual stomach. Furthermore, if possibly tumor-positive lymphatic vessels are to be resected with the primary lesion, the resection area would become even wider. On the contrary, deformity of the residual stomach can be minimized in NEWS, which is one of the distinctive advantages of NEWS, because the operators can optimally and minimally delineate the resection area. Although NEWS may be more complicated and timeconsuming than CLEAN-NET, it would provide more merits for patients than CLEAN-NET.

There are several limitations to this study. Firstly, this was an animal study in a small number of cases on each condition. Secondly, the observational period was relatively short, although 1 week was thought to be enough to verify whether the pigs die from necrosis of the stomach from this procedure. Thirdly, a single tracer method was performed to detect SNs. Currently, a dual tracer method with a radiolabeled material and a dye is clinically recommended for the accurate detection of SNs. Finally, participating operators were limited. Further investigation with various endoscopists/laparoscopists will be needed.

In conclusion, we elucidated the feasibility and safety of NEWS with SNBD in a porcine survival study. This method may provide patients with possibly node-positive EGC a minimally-sized local resection and minimally-ranged lymph node dissection without the risk of tumor dissemination.

Acknowledgments O.G. was funded by a Grant-in-Aid for Young Scientists from The Ministry of Education, Culture, Sports, Science and Technology in Japan in 2012-2013 (Grant No. 25860558). 
Conflict of interest No conflict of interest exists.

\section{References}

1. Ono H, Kondo H, Gotoda T, Shirao K, Yamaguchi H, Saito D, et al. Endoscopic mucosal resection for treatment of early gastric cancer. Gut. 2001;48:225-9.

2. Yamamoto H, Kawata H, Sunada K, Sasaki A, Nakazawa K, Miyata T, et al. Successful en-bloc resection of large superficial tumors in the stomach and colon using sodium hyaluronate and small-caliber-tip transparent hood. Endoscopy. 2003;35:690-4.

3. Oyama T, Tomori A, Hotta K, Morita S, Kominato K, Tanaka M, et al. Endoscopic submucosal dissection of early esophageal cancer. Clin Gastroenterol Hepatol. 2005;3:S67-70.

4. Yahagi N, Uraoka T, Ida Y, Hosoe N, Nakamura R, Kitagawa Y, et al. Endoscopic submucosal dissection using the Flex and the Dual knives. Tech Gastroint Endosc. 2011;13:74-8.

5. Gotoda T, Yanagisawa A, Sasako M, Ono H, Nakanishi Y, Shimoda $\mathrm{T}$, et al. Incidence of lymph node metastasis from early gastric cancer: estimation with a large number of cases at two large centers. Gastric Cancer. 2000;3:219-25.

6. Japanese Gastric Cancer Association. Japanese gastric cancer treatment guidelines 2010 (ver. 3). Gastric Cancer. 2011; 14:113-23.

7. Kitagawa Y, Takeuchi H, Takagi Y, Natsugoe S, Terashima M, Murakami N, et al. Sentinel node mapping for gastric cancer: a prospective multicenter trial in Japan. J Clin Oncol. 2013; 31:3704-10.

8. Takeuchi H, Kitagawa Y. New sentinel node mapping technologies for early gastric cancer. Ann Surg Oncol. 2013;20:522-32.

9. Hiki N, Yamamoto Y, Fukunaga T, Yamaguchi T, Nunobe S, Tokunaga $\mathrm{M}$, et al. Laparoscopic and endoscopic cooperative surgery for gastrointestinal stromal tumor dissection. Surg Endosc. 2008;22:1729-35.
10. Abe N, Takeuchi H, Yanagida O, Masaki T, Mori T, Sugiyama $\mathrm{M}$, et al. Endoscopic full-thickness resection with laparoscopic assistance as hybrid NOTES for gastric submucosal tumor. Surg Endosc. 2009;23:1908-13.

11. Nakajima K, Nishida T, Takahashi T, Souma Y, Hara J, Yamada $\mathrm{T}$, et al. Partial gastrectomy using natural orifice translumenal endoscopic surgery (NOTES) for gastric submucosal tumors: early experience in humans. Surg Endosc. 2009;23:2650-5.

12. Goto O, Mitsui T, Fujishiro M, Wada I, Shimizu N, Seto Y, et al. New method of endoscopic full-thickness resection: a pilot study of non-exposed endoscopic wall-inversion surgery in an ex vivo porcine model. Gastric Cancer. 2011;14:183-7.

13. Mitsui T, Goto O, Shimizu N, Hatao F, Wada I, Niimi K, et al. Novel technique for full-thickness resection of gastric malignancy: feasibility of nonexposed endoscopic wall-inversion surgery (NEWS) in porcine models. Surg Laparosc Endosc Percutan Tech. 2013;23:e217-21.

14. Mitsui T, Niimi K, Yamashita H, Goto O, Aikou S, Hatao F, et al. Non-exposed endoscopic wall-inversion surgery as a novel partial gastrectomy technique. Gastric Cancer. 2013 [Epub ahead of print].

15. Abe N, Takeuchi H, Ohki A, Yanagida O, Masaki T, Mori T, et al. Long-term outcomes of combination of endoscopic submucosal dissection and laparoscopic lymph node dissection without gastrectomy for early gastric cancer patients who have a potential risk of lymph node metastasis. Gastrointest Endosc. 2011;74:792-7.

16. Bok GH, Kim YJ, Jin SY, Chun CG, Lee TH, Kim HG, et al. Endoscopic submucosal dissection with sentinel node navigation surgery for early gastric cancer. Endoscopy. 2012;44:953-6.

17. Inoue $H$, Ikeda $H$, Hosoya $T$, Yoshida A, Onimaru M, Suzuki M, et al. Endoscopic mucosal resection, endoscopic submucosal dissection, and beyond: full-layer resection for gastric cancer with nonexposure technique (CLEAN-NET). Surg Oncol Clin N Am. 2012;21:129-40. 